\title{
INTRAVENOUS THROMBOLYSIS FOR EARLY RECURRENT ISCHEMIC STROKE ASSOCIATED WITH AORTIC ARCH THROMBUS

\author{
T. Karapanayiotides ${ }^{1}$, I. Koutroulou1 ${ }^{1}$, V. Rafailidis ${ }^{2}$, P. Fotiadis ${ }^{1}$, E. Psoma ${ }^{2}$, K. Kouskouras ${ }^{2}$, \\ G. Tsivgoulis ${ }^{3}$, N. Grigoriadis ${ }^{1}$ \\ ${ }^{1}$ Aristotle University of Thessaloniki, 2nd Department of Neurology, Thessaloniki, Greece \\ ${ }^{2}$ Aristotle University of Thessaloniki, Department of Radiology, Thessaloniki, Greece \\ ${ }^{3}$ National \& Kapodistrian University of Athens, 2nd Department of Neurology, Athens, Greece
}

Background \& Aims: Aortic arch atheromatosis proximal to the ostium of the left subclavian artery has been established as a risk factor for recurrent stroke. Safety data of intravenous thrombolysis (IVT) in presence of aortic arch thrombus is extremely scarce ${ }^{1}$. We present the first patient with recurrent cerebral ischemia associated with aortic arch thrombus, treated safely and effectively with IVT.

Case Description: A 51-year-old woman was admitted with a transient episode of expressive aphasia. Apart from smoking her medical history was unremarkable. The neurologic examination was normal and the ABCD2 score was 2. Brain MRI revealed minor acute cortical infarctions in the left middle cerebral artery (MCA) territory (Figure 1, A-C) and no pathology of the intracranial and extracranial vessels. She was started on aspirin. Transthoracic echocardiography and 48-hour holter monitoring were unrevealing. Five days after admission she suffered from occlusion of the left MCA (NIHSS-score:23), confirmed by bedside transcranial Doppler. Brain CT showed only the known minor infarctions. Since mechanical thrombectomy (MT) was not available on a 24/7 basis, we proceeded to off-label IVT with alteplase, starting 80 minutes after the onset of symptoms and resulting within 24 hours to nearcomplete reversal of the neurological deficit (NIHSS-score:1, moderate aphasia). Repeat MRI showed a recurrent infarction in the left posterior MCA territory (Figure 1, D). CT angiography of the aortic arch revealed a voluminous floating thrombus (Figure 2, A-B). She was discharged on acenocoumarol and CT angiography 40 days later documented complete dissolution of the thrombus and a small subjacent ulcerated atheroma (Figure 2, C-F). She was switched to aspirin and high-intensity statin therapy and one year later she suffers only from mild expressive aphasia.

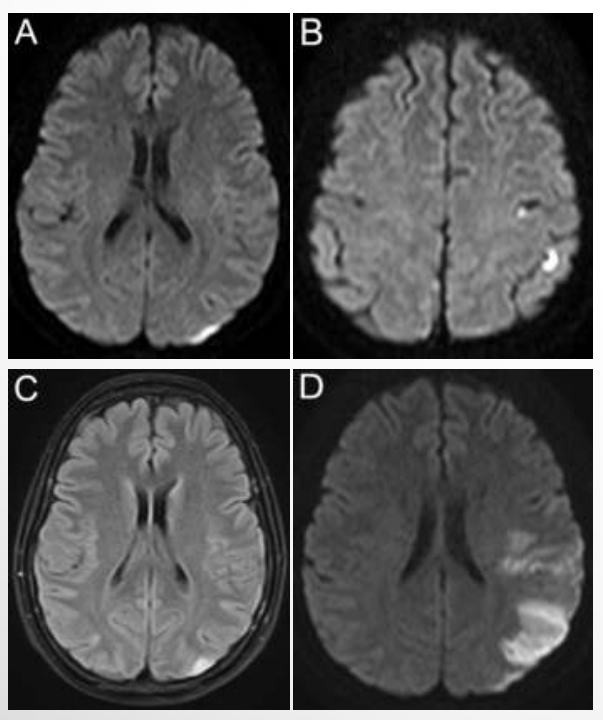

Figure 1. Axial brain DWI showing minor foci of restricted diffusion in the parietal cortical territory of the left MCA (A-B). There is total matching of the lesion size between $D W I$ and (FLAIR) images (C). Repeat axial brain DWI on the sixth day of hospitalization

showing a recurrent infarction in the posterior left MCA territory (D).

1. Krüger $\mathrm{T}$, Liske $\mathrm{B}$, Ziemer $\mathrm{S}$, et al. Thrombolysis to treat thrombi of the aortic arch. Clin Appl Thromb Hemost 2011;17:340-345.
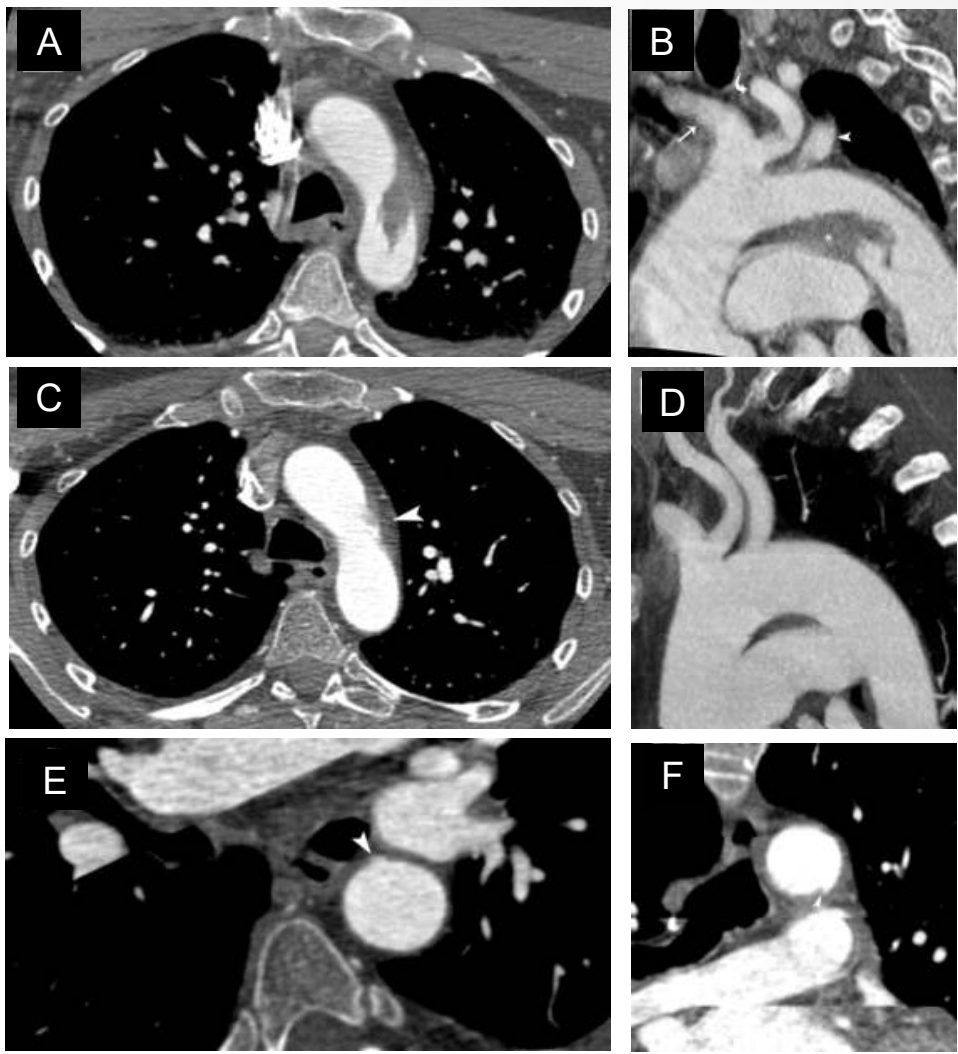

Figure 2. A. Axial CTA showing the free floating thrombus as a flame-like filling defect attached to the wall of the aortic arch. B. The broad-based thrombus (asterisk) is situated at the inferior wall of the aortic arch, facing the origin of the great vessels. Notice the origin of the left common carotid artery (curved arrow) from the brachiocephalic trunk (arrow), so-called "bovine arch", and the origin of the left subclavian artery (arrowhead). C-D. Follow-up CTA after anticoagulation documents complete resolution of the thrombus. Notice the mild subjacent atheroma (arrowhead) that nested the thrombus and the outpouching of the contrast corresponding to an ulceration (E-F).

\section{Conclusions:}

- This is the second case of IVT in a setting of stroke attributed to AAT and the first case of early recurrent stroke treated safely and effectively with IVT in presence of AAT.

- If imaging of the aortic arch is not performed, patients with aortic arch atheromatosis may be mischaracterized as patients with embolic stroke of undetermined source (ESUS).

- Safety of MT and IVT in presence of a mobile aortic arch thrombus is unknown but both treatments may be considered in severely disabling strokes.

- Patients with small infarcts and mild/no deficits after an initial stroke might be considered for IVT in case of early (<3 months) recurrence. 